\title{
Joint transform correlator with spatial code division multiplexing
}

\author{
Zeev Zalevsky, Avi Rubner, Javier García, Pascuala Garcia-Martinez, Carlos Ferreira, \\ and Emanuel Marom
}

\begin{abstract}
A joint transform correlator may suffer from overlapping of the zero diffraction order of the output, which does not contain relevant information, and the correlation peaks that appear in the first diffraction orders if objects are not sufficiently separated. Such overlapping significantly reduces the signal-to-noise ratio of the identification process. We propose a novel approach based on code division multiplexing technique in which the contrast of the identification peaks is significantly enhanced. The approach does not include placing the two objects side by side but rather includes code multiplexing them. Moreover, the code division multiplexing technique allows the space-bandwidth product to be improved. Optical implementation results are given. (C) 2006 Optical Society of America

OCIS codes: $\quad 070.2580,070.2590,070.5010$.
\end{abstract}

\section{Introduction}

Pattern recognition consists of the detection and identification of a known pattern or target in an unknown input scene, which may or may not contain the target, and the determination of the spatial location of any such target. Traditional digital pattern recognition techniques require massive computation and can be relatively slow. Optical techniques can provide inherent parallelism, ultrahigh processing speed, noninterfering communication, and massive interconnection capability and sometimes offer a significantly better alternative to the digital pattern recognition approach. ${ }^{1,2}$

Most optical pattern recognition techniques involve either the use of a matched-filter-based correlator ${ }^{3}$ or a joint transform correlator 4 (JTC). A matchedfilter-based correlator uses Fourier domain complex filter synthesis, whereas a JTC utilizes spatial domain filter synthesis. It is difficult to implement the matched-filter-based correlator for real-time applications because a complex filter must be synthesized

Z. Zalevsky (zalevsz@eng.biu.ac.il) is with the School of Engineering, Bar-Ilan University, Ramat-Gan 52900, Israel. A. Rubner and E. Marom are with the Faculty of Engineering, Tel Aviv University, 69978 Tel Aviv, Israel. J. García, P. Garcia-Martinez, and C. Ferreira are with the Departmento de Óptica, Universitat de València, c/Dr. Moliner 50, 46100 Burjassot, Spain.

Received 10 January 2006; revised 20 April 2006; accepted 21 June 2006; posted 22 June 2006 (Doc. ID 67016).

$0003-6935 / 06 / 287325-09 \$ 15.00 / 0$

C 2006 Optical Society of America and the filter alignment along the optical axis is critical. On the other hand, a JTC is inherently suitable for real-time matching and tracking operations because no complex filter is needed, ${ }^{5}$ as JTC allows both patterns (input and reference) to be displayed side by side, avoiding the need for generating the Fourier transform of the reference pattern. ${ }^{4}$ The JTC realizes the correlation in two cycles. In the first, an optical Fourier transform is realized, and its intensity (joint power spectrum, JPS) is captured by a digital medium. The second cycle includes additional optical Fourier transforming of the captured intensity distribution. Now at the output three diffraction orders are obtained. The zero order contains no relevant terms, while the others are the correlation between the two patterns placed side by side.

One of the main problems associated with the classic JTC is the presence of a strong zero-order peak in the output plane that corresponds to the sum of the autocorrelation terms of the reference and the input signals and almost overshadows the desired correlation peaks.6,7 For a single noise-free target, for example, the zero-order peak is at least four times stronger than the cross-correlation peaks. Javidi and $\mathrm{Kuo}^{8}$ proposed a binary JTC where the JPS was binarized according to a hard-clipping nonlinearity in the Fourier plane. When compared with the classic JTC, a binary JTC is found to yield a superior correlation peak intensity, correlation width, and discrimination sensitivity. Another technique is based on an amplitude-modulated filter, ${ }^{9}$ where the JPS is multiplied by the amplitude-modulated filter before in- 


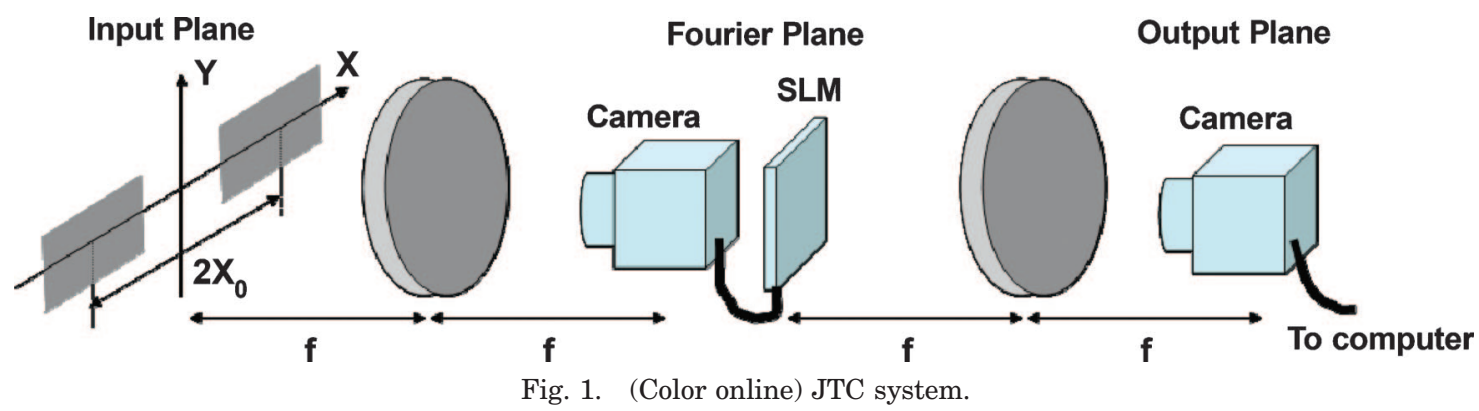

verse Fourier transformation to produce high optical gain for cross-correlation terms. Alam and Karim ${ }^{10}$ proposed a fringe-adjusted JTC in which a realvalued filter called a fringe-adjusted filter is used. The performance of the fringe-adjusted filter is to produce $\delta$ functions as correlation peaks, thus improving the correlation output.

The use of phase spatial light modulators has been applied to JTC implementations ${ }^{11-14}$ to convert amplitude distributions to phase distributions in both the JPS 11 and the joint input plane. ${ }^{12,13}$ It should be noted that, since the present spatial light modulators are capable, under certain conditions, of performing either a phase modulation or an amplitude modulation, JTC systems based only on phase modulation (phase-only systems) that can be found in the entrance or its conjugate planes have been proposed. Even though, because of the CCD camera, this method causes replications after the square part of the correlation points is taken, there is still significant improvement in the energy efficiency at those points. In spite of the improvements obtained with these processors, a spatial light modulator working in the phase regime is not an easy goal. Moreover, the low phase dynamic range modulation is not enough to produce satisfactory results.

We propose a novel approach based on code division multiple access ${ }^{15}$ (CDMA) in which the contrast of the identification peaks is significantly enhanced. CDMA technology focuses primarily on the direct sequence method of spectrum spreading. ${ }^{16} \mathrm{~A}$ direct sequence is spread-spectrum technology in which the bandwidth of a signal is enlarged by artificially increasing the bit data rate by breaking each bit into a number of subbits called chips. The signal is divided into smaller bits by multiplying it by a pseudonoise code. A simple multiplication of the original modulated signal by this high-data-rate pseudonoise code yields the division of the signal into smaller bits, which increases its bandwidth. The bandwidth is expanded by increasing the number of chips. The CDMA technique has been applied to invariant pattern recognition ${ }^{15}$ and to improve the resolution in optical image processors. ${ }^{17,18}$

We use the CDMA approach to increase the bandwidth of JTC systems. In addition, we show that the discrimination capabilities are improved. Instead of placing the objects side by side, we use a vertical interlacing technique to codify the information of the input and the reference into a single amplitude distribution. By choosing the odd or the even pixels in the output plane, we select the zero diffraction order or the first order separately. Optical experimental results are provided by using a spatial light modulator in the amplitude configuration.

In Section 2 we present the description of the proposed approach. Sections 3 and 4 deal with the numerical and the experimental results, respectively. The manuscript is concluded in Section 5.

\section{Description of the Method}

\section{A. Joint Transform Correlator Approach}

The well-known $4-f$ processor $^{3}$ is implemented by means of two lenses having the same focal length $(f)$ separated by a distance of $2 f$ and with, on the Fourier plane, a transparency containing the Fourier transform of the reference pattern. By projecting light onto an object positioned on the front focal plane of the first lens, we obtain its correlation at the back focal plane of the second lens. This method suffers from sensitivity to changes in scale and rotation of the object. The JTC approach does not require placing a transparency of the Fourier transform of the reference object at the Fourier plane of the $4-f$ system. The method places the reference pattern itself side by side with the input object (see Fig. 1).

Let $f\left(x+x_{o}, y\right)$ and $g\left(x-x_{o}, y\right)$ be the reference and the input scene objects centered at $\left(-x_{o}, 0\right)$ and $\left(x_{o}, 0\right)$, respectively. The JPS is

$$
\begin{aligned}
\operatorname{JPS}(u, v)= & \mid F(u, v) \exp \left(\frac{2 \pi i u x_{0}}{\lambda f}\right)+G(u, v) \\
& \times\left.\exp \left(-\frac{2 \pi i u x_{0}}{\lambda f}\right)\right|^{2} \\
= & |F(u, v)|^{2}+|G(u, v)|^{2}+F^{*}(u, v) \\
& \times G(u, v) \exp \left(-\frac{2 \pi i u 2 x_{0}}{\lambda f}\right)+F(u, v) \\
& \times G(u, v)^{*} \exp \left(\frac{2 \pi i u 2 x_{0}}{\lambda f}\right),
\end{aligned}
$$

where $\lambda$ is the wavelength at the illuminating coherent light, $f$ is the focal length, and $F(u, v)$ and $G(u, v)$ are the Fourier transforms of objects $f$ and $g$, respectively. 


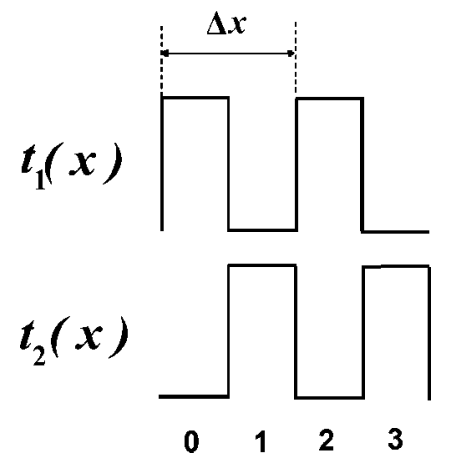

Fig. 2. Masks that block right and left pixels, respectively.

This distribution undergoes additional Fourier transformation by the second lens, and the output distribution equals

$$
\begin{aligned}
C_{\mathrm{JTC}}(x, y)= & f(x, y) * f(x, y)+g(x, y) * g(x, y) \\
& +g(x, y) * f(x, y) \otimes \delta\left(x-2 x_{0}, y\right) \\
& +f(x, y) * g(x, y) \otimes \delta\left(x+2 x_{0}, y\right),
\end{aligned}
$$

where $\otimes$ denotes convolution and $*$ the correlation. From Eq. (2) the autocorrelation terms are centered at the origin, whereas the cross-correlation terms are centered at $\left( \pm 2 x_{0}, 0\right)$. The disadvantage of this JTC system is the requirement for a high spacebandwidth product (SBP) because both the input and the reference share the same input plane. Moreover, an adequate separation between the object and the scene is needed to avoid overlapping effects in the correlation output plane. Also, this correlator is very sensitive to perturbations in the size and orientation of the input pattern.

\section{B. Suggested Approach (JTC-CDMA Method)}

The proposed improvement of the JTC method is analyzed in this subsection. The proposal is to interlace the input and the reference rather than placing them side by side. For instance, such an interlacing may be obtained if the objects are placed one behind the other with appropriate masking. In this case the two objects $f(x, y)$ and $g(x, y)$ are codified by using the geometry shown in Fig. 2, where $t_{1}(x)$ and $t_{2}(x)$ are the masks used for the codification. (See also Fig. 3.) We have considered vertical inter-

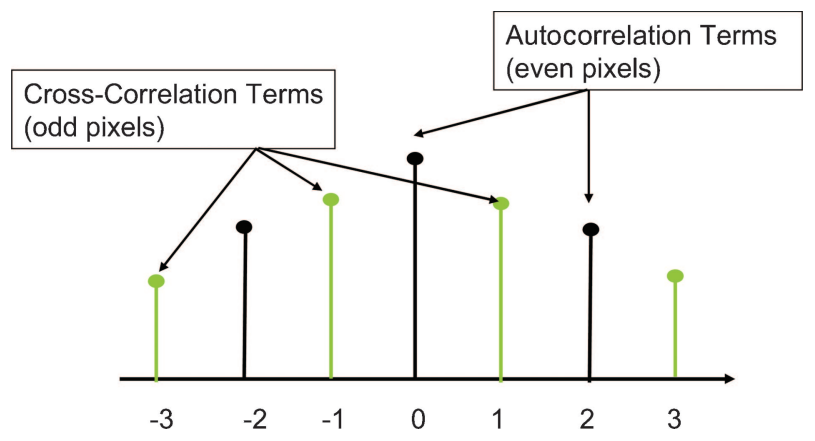

Fig. 4. (Color online) Cross section of the output.

lacing, so the mask has only an $x$-axis dependence. Note that $f(x, y)$ information is codified in the even pixels and $g(x, y)$ in the odd pixels. Moreover, we suppose that the functions are locally smooth enough to preserve the information. The interlacing technique can be viewed as a sampling method. The masks can be written as sampling functions

$$
t_{1}(x)=\sum_{n} \delta(x-n \Delta x), \quad t_{2}(x)=\sum_{n} \delta\left(x-n \Delta x+\frac{\Delta x}{2}\right)
$$

where $\Delta x$ is the interval of sampling and we consider $1 \mathrm{D}$ signal analysis for simplicity. The interlaced distribution displayed in the JTC input plane is written as

$$
s(x)=f(x) t_{1}(x)+g(x) t_{2}(x) .
$$

Once the JPS is registered and a second Fourier transform is performed, the JTC output is

$$
\begin{aligned}
C_{\text {JTC-CDMA }}(x)= & {\left[f(x) t_{1}(x)\right] *\left[f(x) t_{1}(x)\right] } \\
& +\left[g(x) t_{2}(x)\right] *\left[g(x) t_{2}(x)\right] \\
& +\left[g(x) t_{2}(x)\right] *\left[f(x) t_{1}(x)\right] \\
& +\left[f(x) t_{1}(x)\right] *\left[g(x) t_{2}(x)\right] .
\end{aligned}
$$

From Eq. (5) we observe that the first and second terms on the right are the autocorrelations, whereas the third and the fourth provide the cross correlations. In the following we analyze each term sepa-
Input Plane

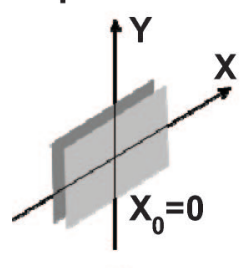

Fourier Plane

SLM

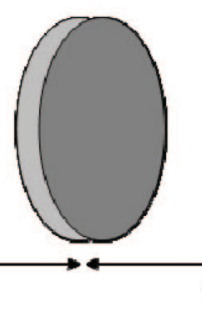

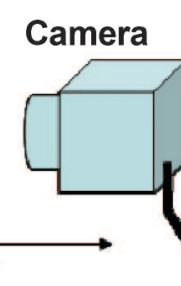

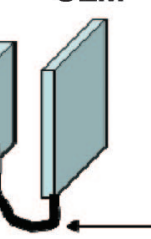

Output Plane

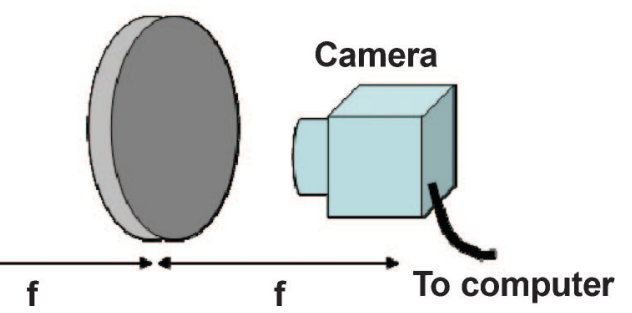

Fig. 3. (Color online) JTC-CDMA system. 

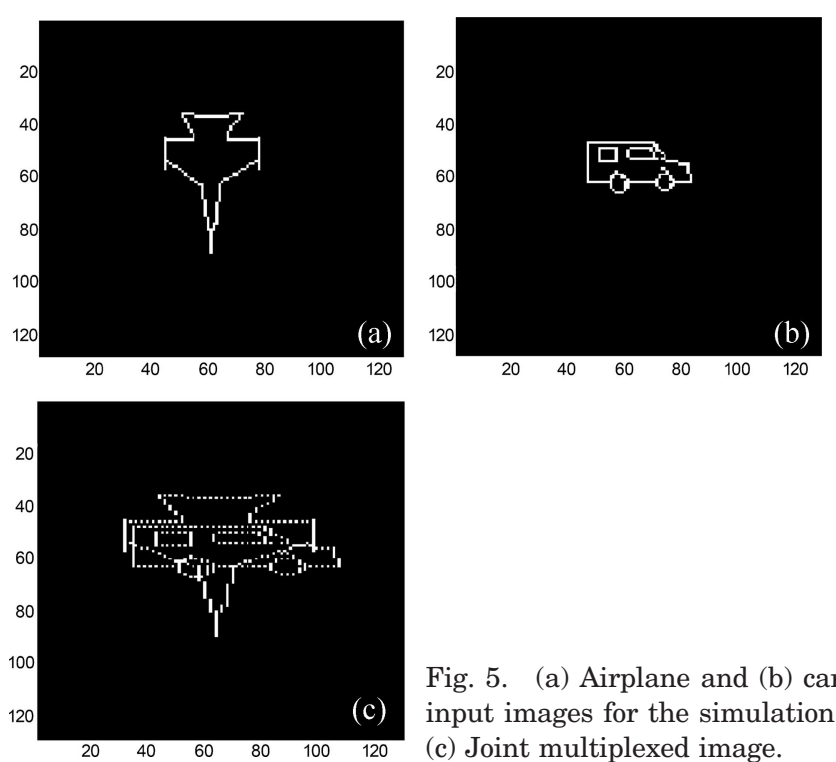

Fig. 5. (a) Airplane and (b) car input images for the simulation. (c) Joint multiplexed image.

rately. The first addend is

$$
\begin{aligned}
{\left[f(x) t_{1}(x)\right] *\left[f(x) t_{1}(x)\right]=} & {\left[f(x) \sum_{n} \delta(x-n \Delta x)\right] * } \\
& {\left[f(x) \sum_{n^{\prime}} \delta\left(x-n^{\prime} \Delta x\right)\right] } \\
= & {[f(x) * f(x)] \sum_{n} \delta(x-n \Delta x), }
\end{aligned}
$$

where we have applied $\delta$ function properties. In the same manner, the second term of Eq. (5) can be written as

$$
\begin{aligned}
{\left[g(x) t_{2}(x)\right] *\left[g(x) t_{2}(x)\right]=} & {\left[g(x) \sum_{n} \delta\left(x-n \Delta x+\frac{\Delta x}{2}\right)\right] * } \\
& {\left[g(x) \sum_{n^{\prime}} \delta\left(x-n^{\prime} \Delta x+\frac{\Delta x}{2}\right)\right] } \\
= & {[g(x) * g(x)] \sum_{n} \delta(x-n \Delta x) . }
\end{aligned}
$$

Equations (6) and (7) show that the autocorrelation will be different from zero at the even pixels of the output. Note that at the origin the information is different from zero; however, in a $\Delta x / 2$ displacement (odd pixels) the autocorrelation is zero.

Analogously, one of the cross-correlation terms can be written as

$$
\begin{aligned}
{\left[g(x) t_{2}(x)\right] *\left[f(x) t_{1}(x)\right]=} & {\left[g(x) \sum_{n} \delta\left(x-n \Delta x+\frac{\Delta x}{2}\right)\right] * } \\
= & {\left[f(x) \sum_{n^{\prime}} \delta\left(x-n^{\prime} \Delta x\right)\right] } \\
& \times \sum_{n} \delta\left(x-n \Delta x+\frac{\Delta x}{2}\right) .
\end{aligned}
$$

Note that the cross correlation will be different from zero only at the odd pixels, whereas at the origin $(x=0)$ the product of $g(x)$ and $f(x)$ is zero. The same result is obtained for the fourth term of Eq. (5). We present Fig. 4 for the sake of clarity.

One of the advantages of this method is a low SBP in the input plane. In addition, there is no longer a need for spatial light modulator with a large SBP in the Fourier plane.

\section{Computer Simulations}

\section{A. JTC-CDMA: Cross Correlation of Two Different Objects}

Two input images of $128 \times 128$ pixels were chosen. The images are a car and an airplane as shown in Figs. 5(a) and 5(b), respectively. Each of the images was coded with the proper mask [Fig. 5(a) is coded with mask $t_{1}(x)$, and the image in Fig. 5(b) with $t_{2}(x)$ ]. We show the JTC-CDMA multiplexed input in Fig. $5(c)$. The two images were interlaced in the $x$ axis such that the one image was represented by the odd and one by the even pixels of the resulting image.

In Fig. 6 we show the (irrelevant) JTC-CDMA output where four correlation terms are involved [see Eq. (5)]. Selecting the even pixels of Fig. 6, we obtain the autocorrelation terms [see Fig. 7(a)]. On the other side, the cross-correlation terms of Eq. (5) are obtained by selecting the odd pixels from the JTCCDMA output plane [see Fig. 7(b)]. We also show a 1D correlation peak profile at the origin. Note that the simulation confirms the results obtained mathematically.
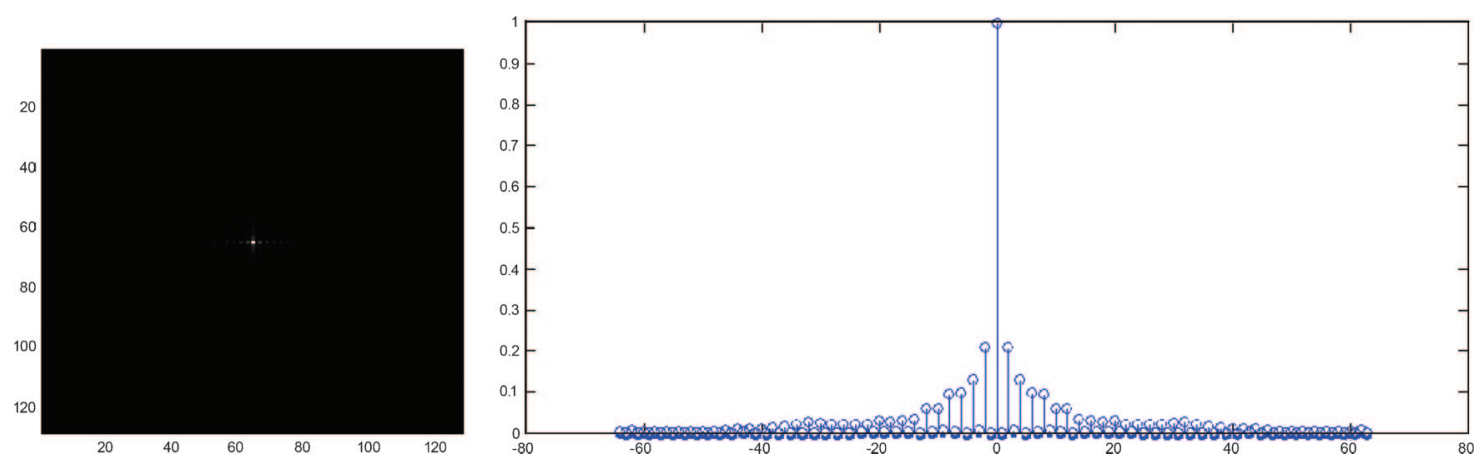

Fig. 6. (Color online) Correlation and its cross-section (airplane and car). 

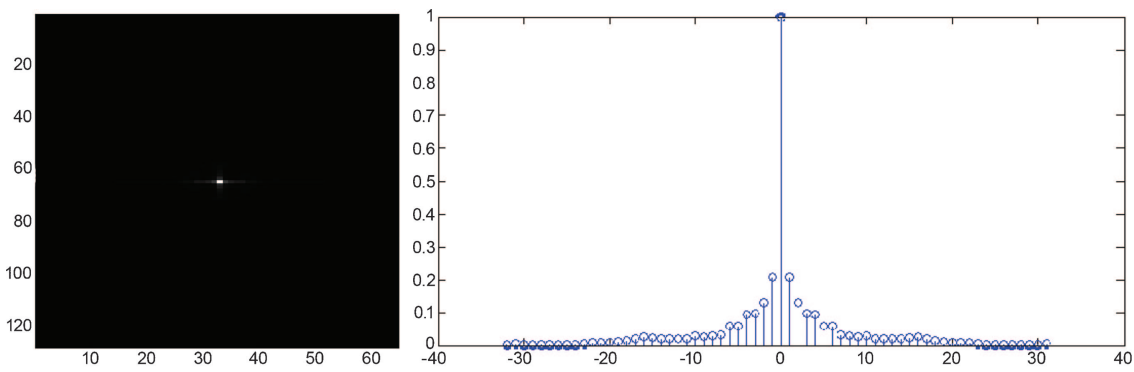

(a)
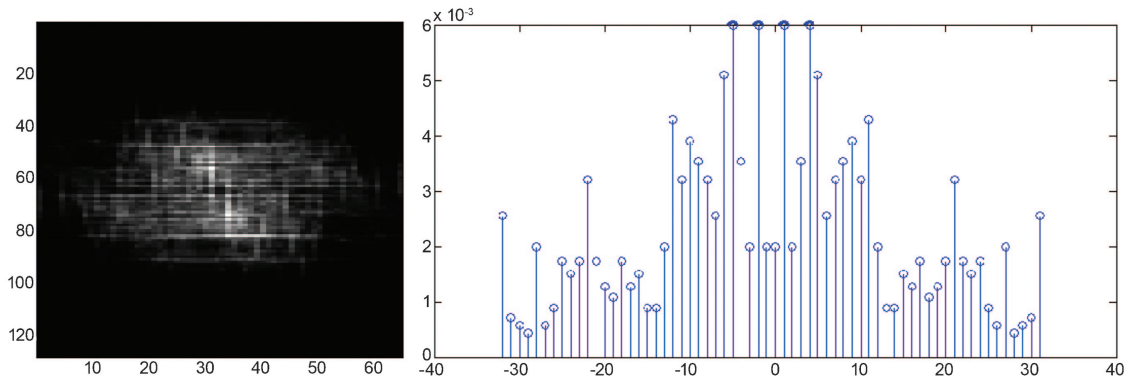

(b)
Fig. 7. (Color online) Simulation results. (a) Autocorrelation and its cross section (even pixels, airplane and car). (b) Cross correlation and its cross section (odd pixels, airplane and car).

\section{B. JTC-CDMA: Autocorrelation of Two Identical Objects} In this simulation identical objects, shown in Figs. $8(\mathrm{a})$ and $8(\mathrm{~b})$, were correlated by using the same approach as in Subsection 3.A. The joint multiplexed image for two identical objects is in Fig. 8(c). In Fig. 9 we show the autocorrelation and cross-correlation results for identical objects when even or odd pixels are selected.

From the simulation results we see that the cross correlation of two different images is very low in comparison with the cross correlation of two identical images (which is in fact the autocorrelation), where a sharp peak is obtained. The ratio between the two peaks in the current images is about 1:75.

\section{Experimental Results}

We have implemented JTC-CDMA optically by using a spatial light modulator operating in amplitude modulation. The spatial light modulator that we use for the optical experiments is an XGA LCTV from CRL Smetic Technology. We calibrated the panel to work as an almost amplitude-only modulator. We worked with a blue laser $(\lambda=473 \mathrm{~nm})$. The corresponding joint power spectra are captured by a CCD camera, and then the second Fourier transformations are performed digitally and optically.

In the first part we show the standard JTC procedure, with no coding. In the input plane we placed an image containing three drawings of cars, two identical and one different, as depicted in Fig. 10. This image went through a Fourier transformation by means of a 2- $f$ system containing a single lens and was captured by a CCD camera connected to a personal computer. We achieved the JPS. After that, we performed on the computer a digitally realized inverse fast Fourier transform (IFFT) and got the correlation image at the output of the JTC, as shown in
Fig. 11. As can be seen, we got diffraction orders of $-1,0,1$, where the 0 order corresponds to the autocorrelation and the -1 and 1 orders correspond to the cross correlation. From the cross sections we find that the ratio between the cross correlation and the autocorrelation is $1: 18$ (the direction of the cross section is the half-diagonal of the correlation picture). In parallel, instead of doing the digitally realized IFFT, we used an all-optical system. In this setup the joint power spectrum was passed through an additional 2- $f$ system to achieve the correlation shown in Fig. 12. When the output picture is partially seen, one can see the autocorrelation at high power and the cross correlation at low power.

In the second part of the experiment we used the CDMA coding on the images (on the cars from Fig.
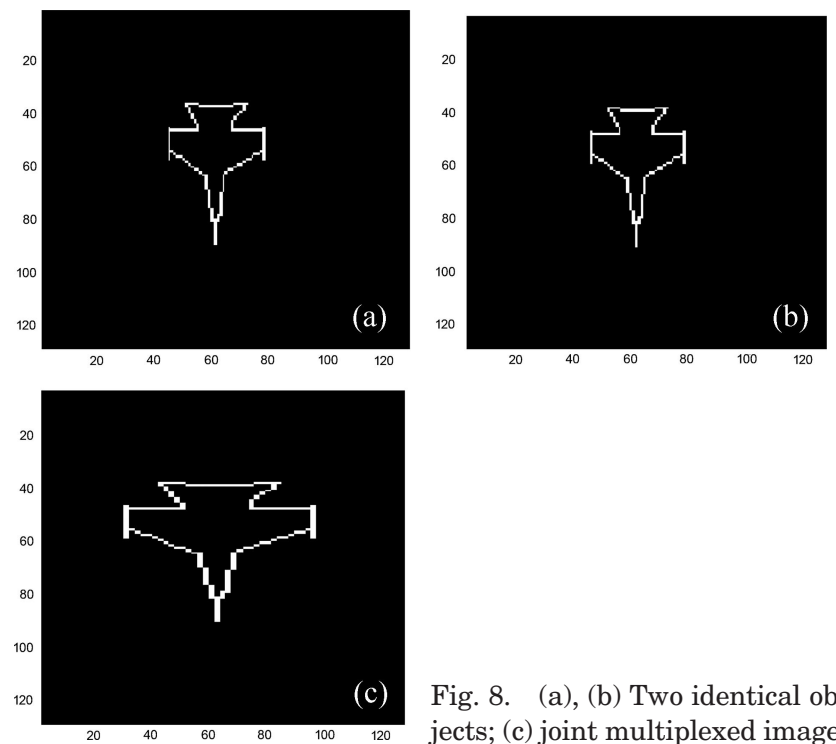

Fig. 8. (a), (b) Two identical objects; (c) joint multiplexed image. 

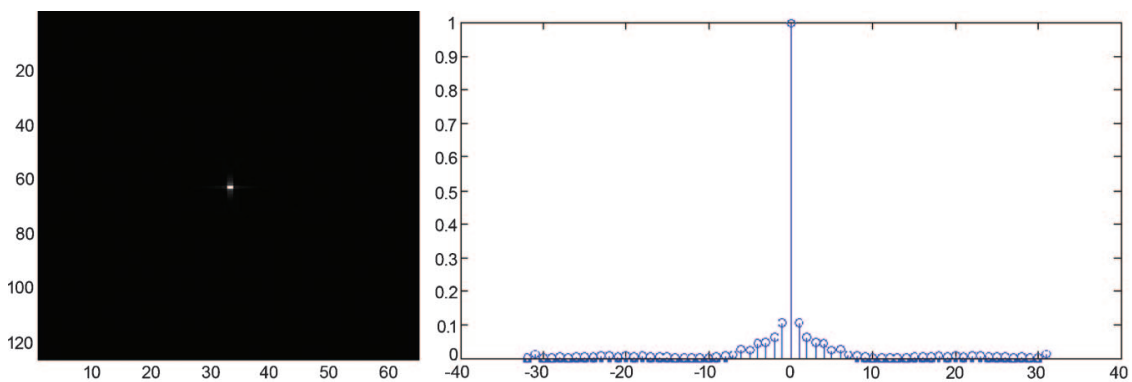

(a)
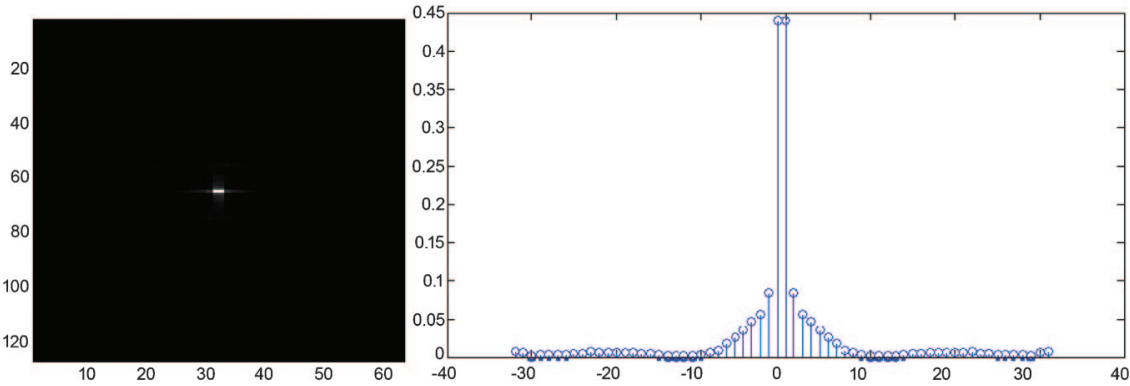

(b)
Fig. 9. (Color online) Simulation results. (a) Autocorrelation and its cross section (even pixels, two airplanes). (b) Cross correlation and its cross section (odd pixels, two airplanes).
10), shown in Fig. 13. The coded images went through a Fourier transform by a 2-f system containing a single lens, as in the first part, which resulted in the spectrum of the image (Fig. 14). On the computer we applied a digitally realized IFFT on the spectrum, which resulted in the correlation image as the JTC output, as shown in Fig. 15 together with the cross section that exhibits a $0.005: 1$ ratio between the cross correlation and the autocorrelation. In Fig. 15(c) we present the results obtained for the even pixels when, instead of the digital IFFT, the second operation was performed optically. However, very careful alignment is required for proper separation of odd and even pixels. One may see the correlation peak observed in the center of the image. This is the autocorrelation peak. The cross-correlation peak obtained for the

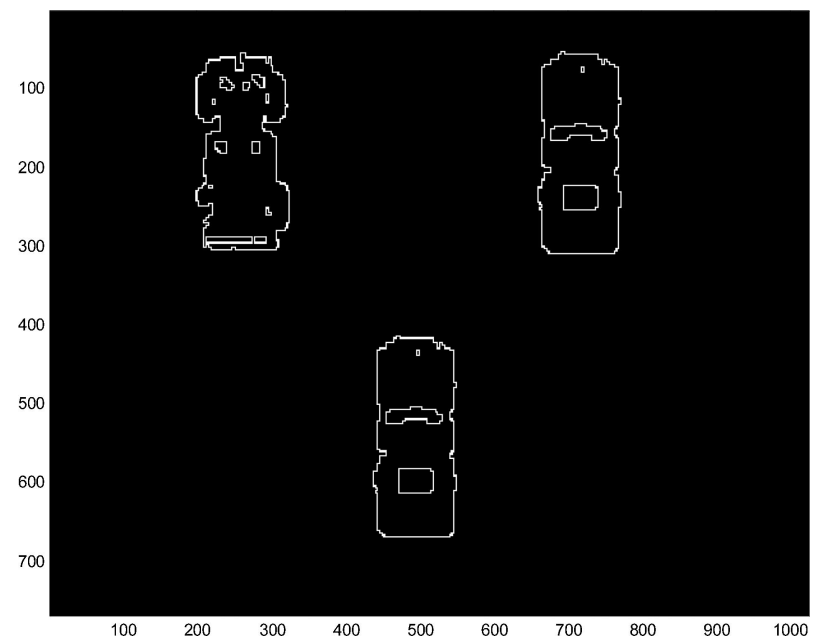

Fig. 10. Input pattern for the regular JTC arrangement.
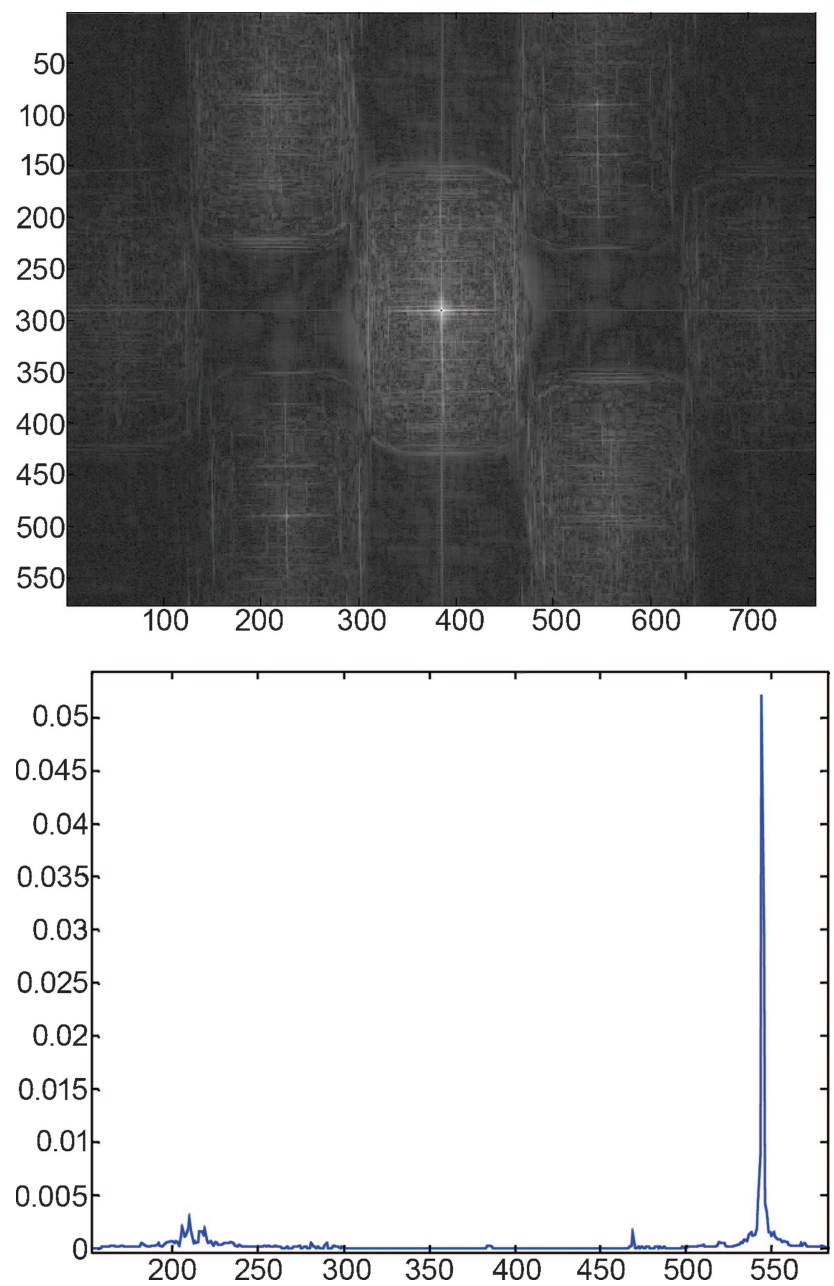

Fig. 11. (Color online) Correlation ratio with half optics and digitally realized IFFT of regular JTC. The ratio between the autocorrelation and the cross correlation was 1:18. 


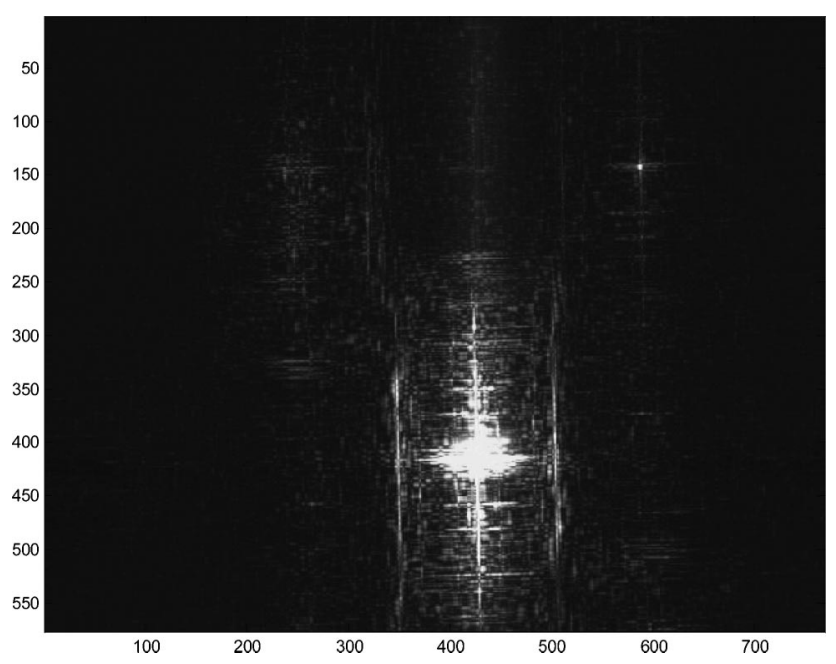

Fig. 12. All-optical experiment; correlation plane of regular JTC.

odd pixels produced a dark image containing only noise. The extinction ratio obtained by using the all-optical method was approximately 0.01:1.

We performed a third experiment in which the goal was to show that a small displacement of the cars from one another (see Fig. 16) in the CDMA image results in a small displacement of the zero-order correlation peak. This occurs because the even pixels of interest contain the autocorrelation and cross correlation. The main reason for this exercise is to obtain better experimental verification by separating the artifacts that are always obtained in the zero order and the peaks coming from the even pixels of the CDMA approach. We wanted to apply the proposed CDMA approach when the even pixels will produce the relevant information but not in the zero order. To do that we generated CDMA multiplexing but also had some spatial separation between the objects. This time we used three (instead of two) objects to produce in the even pixels the cross correlation as well as the autocorrelation (which will be obtained this time in the -1 diffraction order). The multiplexing of the

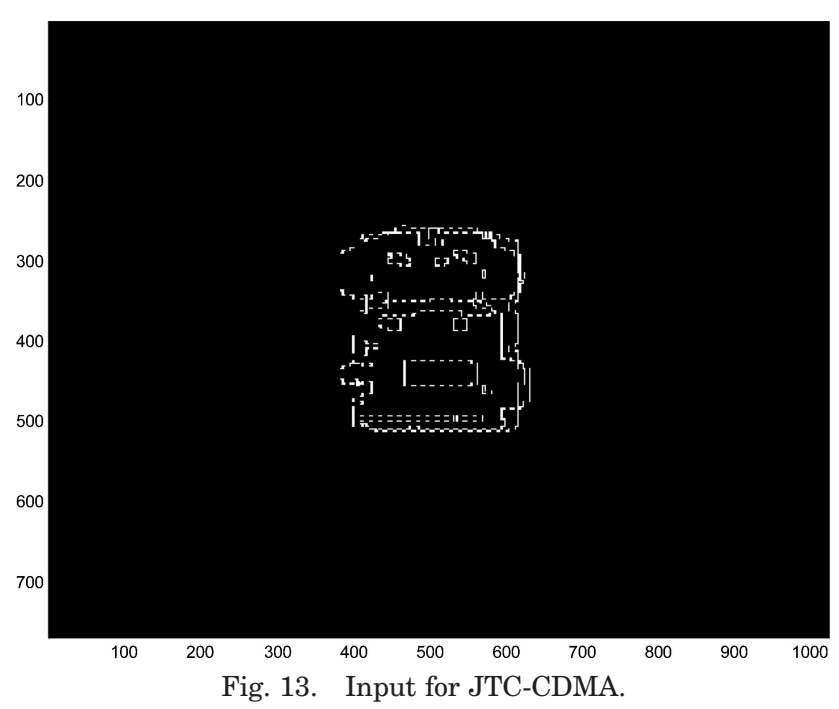

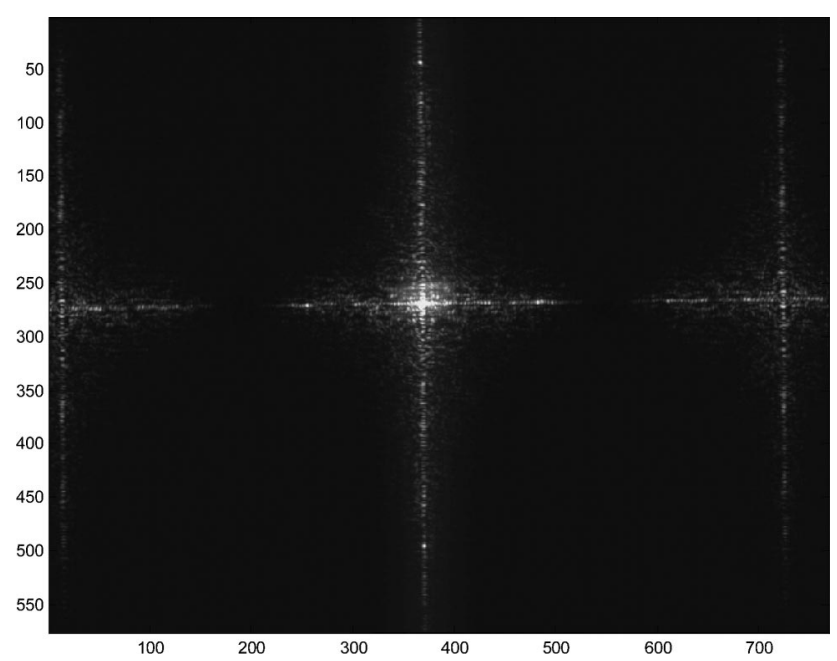

Fig. 14. Joint power spectrum of JTC-CDMA.

objects as seen in Fig. 16 was such that there was a small horizontal shift in addition to a vertical shift, and thus the even pixels are expected to produce the autocorrelation peak, but its position should have a small horizontal shift (to the -1 diffraction order). The cross object has a vertical shift, and thus the cross-correlation peak should appear in the even pixels while it is vertically shifted in comparison with the autocorrelation peak.

In the same manner as the previous experiments, we obtained the spectrum and correlation as in Figs. 17 and 18. The upper part of Fig. 18 presents the
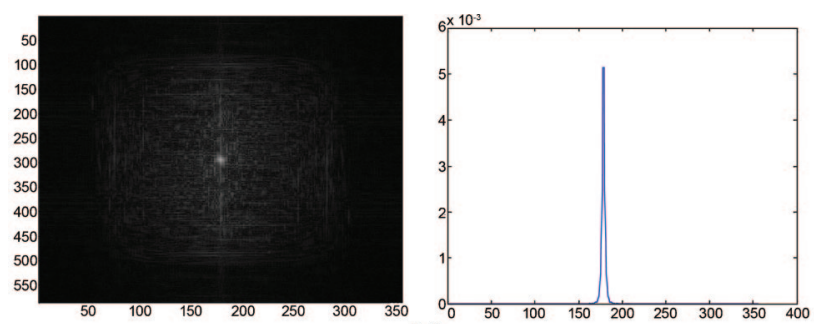

(a)
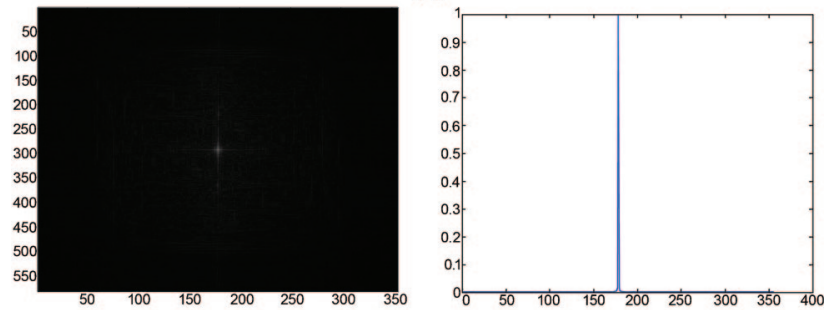

(b)

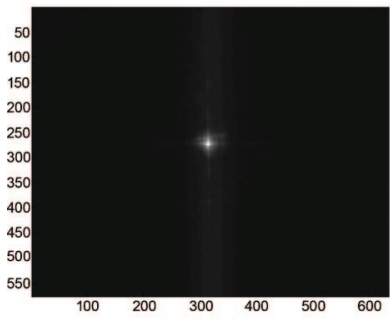

Fig. 15. (Color online) (a) Crosscorrelation output (odd pixels) with the $1 \mathrm{D}$ profile and (b) autocorrelation output (even pixels) with the $1 \mathrm{D}$ profile, both results with digital IFFT. (c) Autocorrelation output (even pixels) with (c) the IFFT performed optically. 


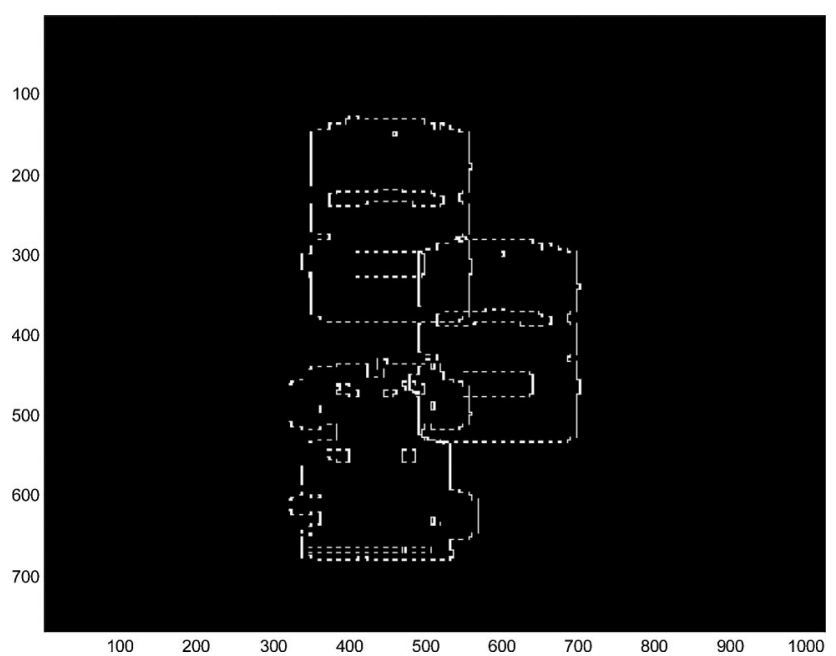

Fig. 16. Input object with a small shift.

experimental result when we conducted the experiment by using a Fourier transform through a $2-f$ system followed by a digitally realized IFFT. The bottom part of Fig. 18 (the cross section) presents the vertical cross section of the left-hand image in the -1 diffraction order (at the coordinates of the upper part of Fig. 18 , it is the plot of all the rows along column number 90). The cross section in the bottom part of Fig. 18 that presents the cross correlation and the autocorrelation shows that the ratio of the autocorrelation and the cross correlation is about $0.02: 1$, which is smaller than what we observed in the other experiment.

Figure 19 presents the output correlation plane of the even pixels after realizing the second Fourier transform (over the JPS) optically rather than by use of digital IFFT. The presented image corresponds to the spatial region around the relevant diffraction order of -1 , where the autocorrelation and crosscorrelation peaks should appear for the even pixels. One may see that the autocorrelation peak obtained around pixel number 130 is much stronger than the

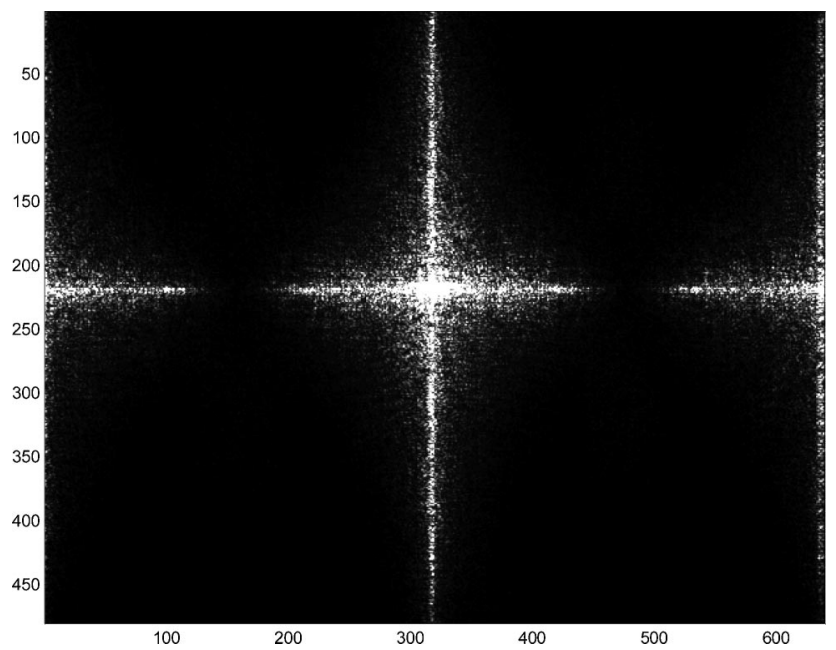

Fig. 17. Joint spectrum of input object with a small shift.
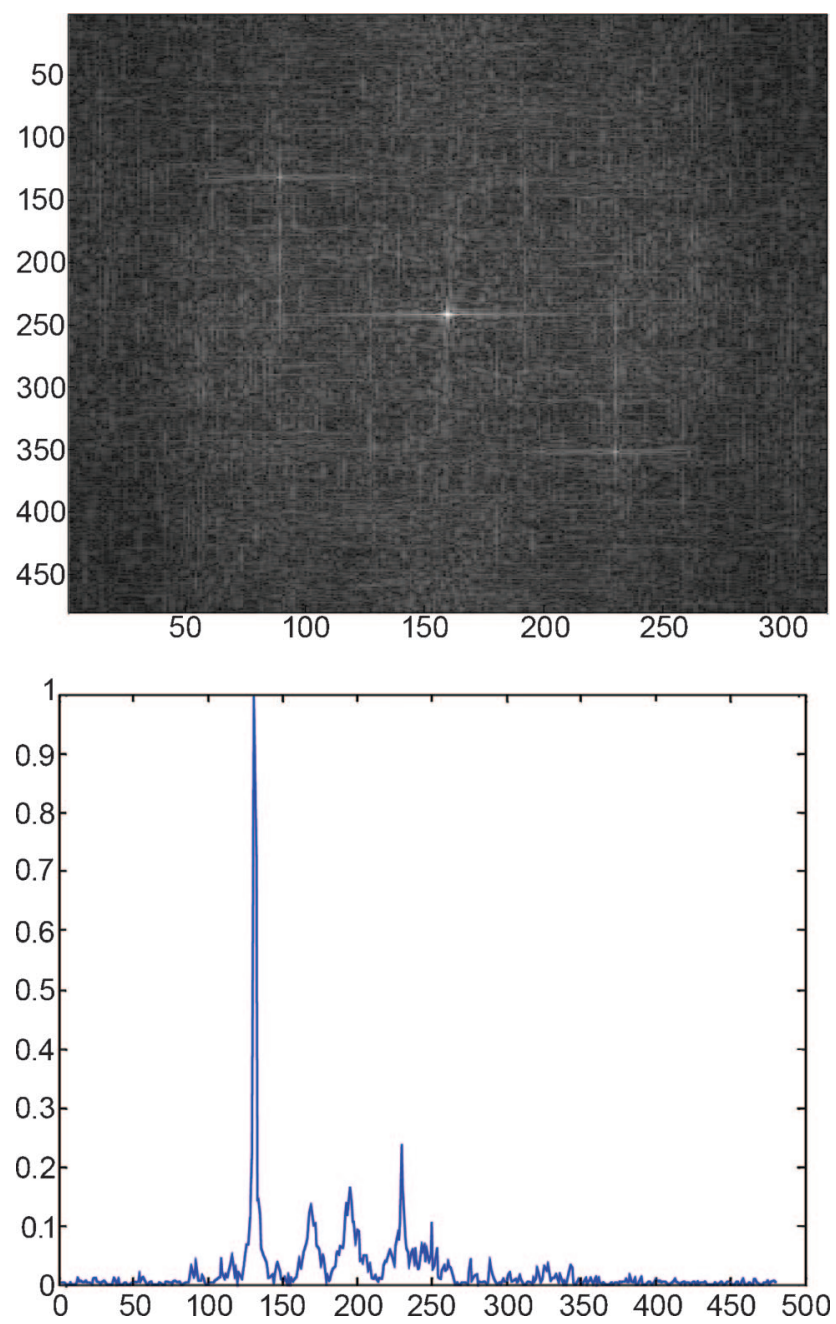

Fig. 18. (Color online) Correlation after digital IFFT and its cross section of the autocorrelation and cross-correlation peaks of the input object with a small shift.

cross-correlation peak that should appear around pixel 340 . The ratio obtained between the two of them was approximately 1:0.025.

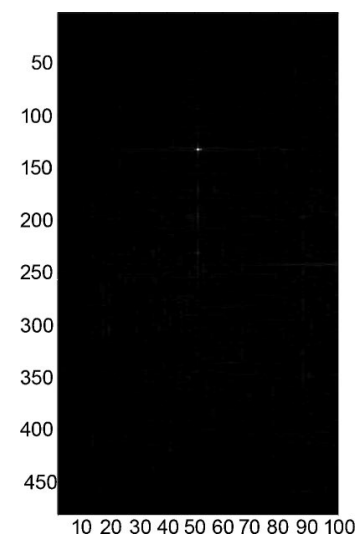

Fig. 19. Correlation plane after optical IFFT of the joint spectrum. The presented region corresponds to the spatial region around the diffraction order of -1 , where the autocorrelation and cross-correlation peaks should appear. 


\section{Conclusions}

We have presented a new method for performing correlations in a JTC system. Traditional JTC systems suffer from the problems of the SBP and low contrast between the autocorrelation and the cross-correlation peaks. Here, instead of placing the objects side by side with a certain distance between them, we code the objects by using code division multiplexing so that we get a single object in the input plane. The experiments show that the new method results in a high contrast of $0.005: 1(1: 200)$ in comparison with the traditional method, where the contrast is $1: 18$. This improvement is significant when similar or different objects are being indentified.

This work was supported by Fondo Europeo de Desarrollo Regional funds and the Spanish Ministerio de Educación y Ciencia under project FIS2004-06947C02-01, the Agencia Valenciana de Ciencia y Tecnología project GRUPOS03/117, the D.G Investigació i Transferència Tecnològica project IIARC0/2004/217.

\section{References}

1. J. W. Goodman, Introduction to Fourier Optics, 2nd ed. (McGraw-Hill, 1996).

2. B. G. Boone, Signal Processing Using Optics: Fundamentals, Devices, Architectures, and Applications (Oxford U. Press, 1998),

3. A. VanderLugt, "Signal detection by complex spatial filtering," IEEE Trans. Inf. Theory IT-10, 139-145 (1964).

4. C. S. Weaver and J. W. Goodman, "A technique for optically convolvings two functions," Appl. Opt. 5, 1248-1249 (1966).

5. P. Purswosumarto and F. T. S. Yu, "Robustness of joint transform correlator versus VanderLugt correlator," Opt. Eng. 36, 2775-2780 (1997).

6. M. S. Alam, A. A. S Awwal, and M. A. Karim, "Improved corre- lation discrimination using joint Fourier transform optical correlator," Microwave Opt. Technol. Lett. 4, 103-106 (1991).

7. F. T. J. Johnson, T. H. Barnes, T. Eiju, T. G. Haskell, and K. Matsuda, "Analysis of a joint transform correlator using a phse-only spatial light modulator," Opt. Eng. 30, 1947-1957 (1991).

8. B. Javidi and C. Kuo, "Joint transform image correlation using a binary spatial light modulator at the Fourier plane," Appl. Opt. 27, 663-665 (1988).

9. D. Feng, H. Zhao, and S. Xia, "Amplitude-modulated JTC for improving correlation discrimination," Opt. Commun. 86, 260-264 (1991).

10. M. S. Alam and M. A. Karim, "Fringe-adjusted joint transform correlator," Appl. Opt. 32, 4344-4350 (1993).

11. F. T. J. Johnson, T. H. Barnes, T. Eiju, T. G. Haskell, and K. Matsuda, Opt. Eng. 30, 1947-1957 (1991).

12. G. Lu, Z. Zhang, and F. T. S. Yu, "Phase-encoded input joint transform correlator with improved pattern discriminability," Opt. Lett. 20, 1307-1309 (1995).

13. G. Lu and F. T. S. Yu, "Performance of the phase-transformed input joint transform correlator," Appl. Opt. 35, 304-313 (1996).

14. P. S. Erbach, D. A. Gregory, and J. B. Hammock, "Phase-only joint transform correlator: analysis and experimental results," Appl. Opt. 35, 3091-3096 (1996).

15. J. Solomon, Z. Zalevsky, D. Mendlovic, and J. García, "Filter multiplexing by use of spatial code division multiple access approach," Appl. Opt. 42, 772-777 (2003).

16. A. J. Viterbi, CDMA, Principles of Spread Spectrum Communication (Addison-Wesley, 1996).

17. J. Solomon, Z. Zalevsky, and D. Mendlovic, "Invariant pattern recognition by use of a spatial code division multiplexing approach,” Appl. Opt. 42, 3345-3355 (2003).

18. J. Solomon, Z. Zalevsky, and D. Mendlovic, "Geometric superresolution by code division multiplexing," Appl. Opt. 44, 32-40 (2005). 